hallucinations and persecutory delusions. Five days later she was overtalkative, very active, and irritable, and ten days later she had mild pressure of speech. She made a good recovery and was discharged on lithium.

It appears that one of Crammer's criteria for linking the menstrual cycle and the mental state has been fulfilled. While amenorrhoeic due to pregnancy, the patient remained well. Three days after birth she relapsed with a similar clinical picture to those episodes linked to the menstrual cycle.

I have now reported the case of a woman with a premenstrual psychotic illness who subsequently suffered from a puerperal relapse. This is complementary to those cases of puerperal psychosis and subsequent premenstrual relapse (Brockington et $a l, 1988)$ and adds substantially to the hypothesis that these phenomena have the same aetiology. One factor common to both the premenstrual and the postnatal period is the change in the sex hormones and it has been argued that there is an interaction between changes in the levels of the sex hormones and dopamine receptor sensitivity (Wieck et al, 1992). Relapse of a puerperal psychosis has also been noted after the removal of a hydatidiform mole, when levels of gonadotrophins, progesterone and also oestrogen would be expected to fall (Hopker \& Brockington, 1991). The circumstantial evidence that puerperal psychotic illness is secondary to falling levels of hormones is considerable. It does, however, remain true that there are a number of different hormones and neurotransmitters that may be responsible for this intriguing interaction.

Brockington, I. F., Kelly, A., Hall, P., et al (1988) Premenstrual relapse of puerperal psychosis. Journal of Affective Disorders, 14, 287-292.

Hopker, S. W. \& Brockington, I. F. (1991) Psychosis following hydatidiform mole in a patient with recurrent puerperal psychosis. British Journal of Psychiatry, 158, 122-123.

WIECK, A., KUMAR, R., HIRST, A. D., et al (1992) Increased sensitivity of dopamine receptors and the recurrence of affective psychosis after childbirth. British Medical Journal, 303, 613-616.

Institute of Psychiatry

SiMON LOVESTONE

De Crespigny Park

Denmark Hill

London SE5 8AF

\section{Gender differences in schizophrenia}

SIR: Lewis (Journal, October 1992, 161, 445-450) comments that gender differences in schizophrenia are established findings. Iacono \& Beiser (1992) noted the conventional belief is that the incidence of schizophrenia is the same for both sexes. They commented that surveys over the past ten years have provided inconsistent results. They referred to studies in Asia, Europe, and North America, suggesting that an excess of schizophrenia does occur among males.

A prospective assessment of gender differences in psychiatric illness among a sample of 70 patients admitted to a psychiatric unit, in South Africa, showed the following results. The incidence of schizophrenia was more common among men than women $(P=0.003)$. The majority of patients in the 10-30 year age group were men, while women patients predominated in the 30-50 year age group $(P=0.029)$. This study showed that the men presented earlier with mental illness, this tending to be schizophrenia.

Dr Lewis comments on the biological basis for the male predominance. Though sociocultural factors relating to traditional societies may be unique in Africa, it would appear that results from South Africa are consistent with those from the rest of the world.

IACONo, W. G. \& BeIsER, M. (1992) Are males more likely than females to develop schizophrenia? American Journal of Psychiatry, 149, 1070-1074.

\section{Davenport Road}

Catherine M. Maud

\section{Glenwood}

Durban

South Africa 4001

\section{Carbamazepine and episodic dyscontrol}

SIR: Sugarman (Journal, November 1992, 161, 721) reports three individuals with outbursts of violence who responded well to carbamazepine. Interestingly, all had olfactory hallucinations and reported déjà vu experiences. These features point towards a diagnosis of temporal lobe epilepsy despite the absence of electroencephalogram (EEG) changes in two of the cases. Therefore, it is not surprising that the response to anticonvulsants was favourable.

I would agree the episodic dyscontrol may be "best understood as paroxysmal violence, due to epilepsylike dysfunction of limbic structures in the temporal lobe", but this would appear to be a rather unfashionable view. I think it is worth noting that Maletsky's cases (1973) often had long histories of violent behaviour and, despite the 'out of character' quality of the episodes, I suspect many psychiatrists would have diagnosed them as suffering from an antisocial personality disorder.

The DSM-III-R criteria for intermittent explosive disorder have been designed specifically to exclude 
those individuals with 'between episode' behavioural abnormalities to avoid a legalistic haven for habitually violent individuals. It seems difficult to differentiate between episodes of dyscontrol and 'between episode' aggressiveness. The criteria are, however, consistent with the current view that individuals with antisocial personalities are in the main not treatable and, therefore, not part of the work of psychiatrists.

Disappointingly, despite a considerable number of studies showing a reduction of aggressive behaviour linked to anticonvulsant treatment, anticonvulsants have not been widely used in the absence of definite brain damage. I would suggest that some people do have episodes of violent behaviour mediated by limbic dysfunction of some kind, but that they rapidly develop a range of secondary difficulties in the areas of education, employment, relationships, etc. It then becomes impossible to define them as a group, as they appear identical to individuals with antisocial personality disorder.

MALETSKY, B. M. (1973) The episodic dyscontrol syndrome. Diseases of the Nervous System, 34, 178-185.

\section{Rampton Hospital}

Retford

Nottinghamshire DN22 OPD

Simon D. PAYNE

\section{SSRIs and tricyclic antidepressants}

SIR: The case studies of Seth et al (Journal, October 1992, 161, 562-565) suggesting combination studies with 5-HT reuptake inhibitor and noradrenergic tricyclic antidepressant (with or without concurrent lithium therapy) appear to offer new hope for the treatment of resistant depression, especially in elderly patients.

It must be remembered that 5-HT reuptake inhibitors are pharmacologically distinct and a heterogeneous group of drugs. The toxicity of tricyclic antidepressants is plasma-dependent (Preskorn, 1991). Certain 5-HT reuptake inhibitors are known to inhibit tricyclic antidepressant metabolism; paroxetine ( $30 \mathrm{mg}$ per day) causes a three-fold increase in half-life and a five-fold increase in the clearance serum desipramine (Brosen et al, 1992). There are a number of case reports testifying to the clinical relevance of interaction between fluoxetine and tricyclic antidepressant levels (e.g. Brosen \& Skelbo, 1990). Sertraline appears to have less effect in inhibiting the metabolism of tricyclic antidepressants. There is some indication that an antidepressant-free interval may be necessary when switching from fluoxetine to a tricyclic, if one is to be cautious to avoid potentially toxic tricyclic plasma levels (van Ameringen \& Mancini, 1992).

The Committee on Safety of Medicines (1989) warned that the combinations of selected 5-HT reuptake inhibitors with monoamine oxidase inhibitors, lithium, or tryptophan, while potentially beneficial in some selected cases, can increase the severity of 5-HT-related side-effects. In the most severe cases (life-threatening 5-HT syndrome of hypothermia), tremor convulsions may develop.

In the management of elderly depressed people who may often have concomitant physical illness, serious consideration must be given when choosing a selective 5-HT reuptake inhibitor for use in combination therapy, with a clear understanding of the potential risks associated with the various drugs.

Brosen, K., et al (1992) Pharmacogenetics of tricyclic antidepressants and novel antidepressants: recent developments. Clinical Neuropharmacology, 15, 80A-81A.

- \& SKelbo, E. (1990) Fluoxetine and norfluoxetine are potent inhibitors of P450-II-D6 - the source of the sparteine/ debrisoquine oxidation polymorphism. British Journal of Clinical Pharmacology, 32, 136-137.

Committee on SAfeTy of Medicines (1989) Current Problems (May).

Preskorn, S. H. \& FAST, G. A. (1991) Therapeutic drug monitoring for antidepressants: efficacy, safety and cost effectiveness. Journal of Clinical Psychiatry, 52, 23-33.

van Ameringen, M. \& Mancini, C. (1992) Adverse effects of switching from fluoxetine to desipramine. Canadian Journal of Psychiatry, 37, 278.

L. J. ASHWORTH

The University of Liverpool

K. C. M. WILSON

Department of Psychiatry

P.O. Box 147

Liverpool L69 3BX

\section{HTLV-1 Revisited}

SIR: In view of the continuing investigation into the aetiology of psychotic illness, we wish to report our findings on the seroprevalence of human $\mathrm{T}$-cell lymphotropic virus-1 (HTLV-1) in a group of acute psychiatric patients in Trinidad and Tobago.

HTLV-1 is endemic to Trinidad and Tobago with a reported seroprevalence of $1.6 \%$ among the healthy population (Daisley et al, 1991). Retroviral infection has been suggested as a possible aetiological mechanism in psychosis, especially since retroviruses can be incorporated into the human genome (Crow, 1984). Although previous studies have not found a demonstrable link between retroviral infection and schizophrenia (Feenstra et al, 1989), we felt that because HTLV-1 is endemic to our population, the 